\title{
Statistical tyre/road noise modelling based on continuous 3D texture data
}

\author{
Reinhard Wehr ${ }^{1,2, *}$, Andreas Fuchs ${ }^{1}$, and Simon Breuss ${ }^{1}$ \\ ${ }^{1}$ AIT Austrian Institute of Technology, 1210 Vienna, Austria \\ ${ }^{2}$ TU Wien, 1040 Vienna, Austria
}

Received 23 April 2021, Accepted 24 September 2021

\begin{abstract}
In this article, statistical modelling approaches for tyre/road noise levels (according to the CPX method) based on continuous 3D texture measurements are presented. The main focus is to estimate if viable correlations of interpretable 3D texture parameters with frequency-dependent CPX levels can be found. Therefore, a set of descriptive 3D texture parameters is introduced. Two different modelling approaches, focussing on linearity in order to gain interpretability, are shown. First, a linear model is calculated based on the first principal components of the texture parameters, and second, a random forest regression approach is performed on the direct texture parameters. As a principal component analysis of the CPX measurement data reveals three highly correlated frequency ranges, both approaches are calculated independently for low, mid and high frequency bands. Both models show good performance in the low and mid frequency range, whereas the accuracy in the high frequency range declines. Due to the focus on linearity, both approaches result in comparable statistical benchmark parameters.
\end{abstract}

Keywords: Tyre/road noise, CPX method, 3D road surface texture, Statistical modelling

\section{Introduction}

Road noise can be seen as main source of noise pollution in Europe [1]. Fifteen percent of the European population are reported as to be exposed to high levels of road traffic noise during the night-time period, with an even higher percentage of $20 \%$ during the day-evening-night period. In [2], the reduction of road noise to a level of $53 \mathrm{~dB}\left(L_{\mathrm{den}}\right)$ resp. a level of $45 \mathrm{~dB}\left(L_{\text {night }}\right)$ is strongly recommended by both reductions at the source as well as on the transmission path. In [3], the WHO reports an estimated loss of disabilityadjusted life-years of 1.0-1.6 million DALYs in western Europe. This substantiates the need for reducing road noise.

Generally speaking, points of leverage to tackling road noise can be summarized in four categories:

- traffic volumes,

- speed limits,

- noise generation at the source,

- noise propagation/transmission path.

The first two categories can be influenced by legislative and/or political resolutions. For the remaining two categories, technical solutions may help in noise reduction at the source and the propagation path.

*Corresponding author: reinhard.wehr@ait.ac . at
Reducing traffic noise in the propagation path is widely accomplished by the installation of noise barriers adjacent to main traffic corridors. A general overview of the use of noise barriers along European roads can be found in [4].

Reductions at the source may be the most straightforward approach towards noise control. Here, from approx. 40-50 km/h upwards until approx. $130 \mathrm{~km} / \mathrm{h}$ (where noise from air turbulences of the vehicle body begin to dominate), tyre/road noise is the main source for noise generation [5], although this crossover is changing towards lower speeds due to the increase in electric vehicles. Different generation mechanisms, mainly tyre vibrations, aeroacoustic effects such as air pumping, and stick/slip effects, lead to complex frequency-dependent interactions in noise generation. These mechanisms are strongly dependent on the road surface, where the surface macrotexture as well as the void contents for porous and semi-porous road surfaces exhibit an important influence [5].

Measurement methods for determining the rolling noise of different tyres are e.g. described in [6] and performed on a special test road surface [7]. Unfortunately, it is indicated that a direct transfer of the results obtained on such a test track to typical road surfaces may not be possible [8]. For assessing the influence of different road surface types, the standardized measurement methods ISO 11819-1 for passby noise and ISO 11819-2 (CPX method) for the continuous on-board determination of tyre/road noise $[9,10]$ exist. 
A study analyzing the relationships of the two methods can be found in [11]. The CPX method, which was used in the work presented furtheron, is based on a reference test tyre [12] in order to focus on the impact of the road surface on the noise emission. Influences of the tyre and environmental conditions are adjusted by correction procedures, and were recently studied extensively [13-17]. Measurement uncertainties due to the interrelations between tyre and road temperature as well as tyre shore hardness were analyzed in [18], and results of round-robin tests can be found in [19-21].

In order to understand the generation mechanisms of tyre/road noise, approaches to simulate a rolling tyre were performed over the last 20 years. General research on the topic of the tyre/road texture contact with focus on the contact forces by small roughnesses can e.g., be found in $[22,23]$. The influence of the contact stiffness on tyre vibration induced noise was studied in [24] by means of simulations. Approaches to physically model the vibrations of a radiating tyre on an ISO surface are e.g., introduced in [25]. A statistical approach to correlate the contact forces with low-frequency tyre/road noise was performed in [26]. There, short sections of 3-dimensional texture measurements were used to characterize different road surface types and thereby estimate tyre/road noise in one-third octave bands. High levels of the correlation coefficients are reported, whereas the standard deviation is indicated as above $1 \mathrm{~dB}$ below the frequency of $1250 \mathrm{~Hz}$.

In this article, a more direct approach towards statistical modelling of tyre/road noise emissions based on 3D texture measurements is presented. In order to characterize the road surface, a high-resolution texture scanner was used to continuously measure the road surface directly in front of the tyre of a CPX measurement system. Thereby, the actual input texture geometry was measured and furtheron used within the modelling process. Due to the large amount of texture data, the focus of the research work is not on the physical contact modelling, but on the determination of meaningful and interpretable 3D texture parameters in order to estimate the potential of modelling approaches of tyre/road noise based on 3D texture measurements.

\section{Description of the dataset}

The measurement locations used for the modelling approach dataset consist of more than 50 stone mastic asphalt (SMA) sections on Austrian motorways. Information regarding the road surface type as well as its age and traffic loads were merged with the measurement locations. On the measurement sections, typically $500 \mathrm{~m}$ long measurements of CPX levels and 3D texture were performed. All road surfaces used for the analysis were in good condition, viz. not exhibiting cracks or apparent degradation.

In general, no further details regarding the exact road surface type, viz. maximum aggregate size or void content of the SMA, were known. Regarding the maximum aggregate size, nevertheless, with regard to the Austrian building regulations, a value of $11 \mathrm{~mm}$ can be assumed throughout the dataset. Also, this quantity should be implicitely treated by the model, as 3D texture information is its main input parameter. Concerning the void content, SMA surfaces in Austria are categorised in 2 relevant groups (SMA S1 with a void content of 1.5-4.0\% and SMA S3 with a void content of $6.0-12.0 \%$ ). These groups will be treated as one in the modelling process, but will be distinguished throughout this article as to further discuss possible influence factors.

CPX levels were measured and evaluated according to the ISO standard using the P1 [12] tyre. As measurement speed, $60 \mathrm{~km} / \mathrm{h}$ was chosen, as this was the maximum speed of the 3D texture measurement system used. Only the right wheel track and only the rear microphone position was used for the modelling approach. This is due to the 3D texture sensor installed in front of the right measurement tyre. Thereby, it could be ensured that the measured road surface texture worked as the input function into the tyre/road interaction. By choosing the rear microphone, possible influences, viz. acoustic reflections on the sensor, could be diminished. This precaution may not be necessary with regard to the modelling approach, as the measurement system was not altered during the data acquisition, and said reflections can be assumed as a constant additive disturbance altering the measurement levels, but not the modelling approach, as long as the road surface type is kept constant.

The 3D texture measurements were performed with an in-house developed measurement system (see Sect. 2.2, [27]) with a spatial resolution of approx. $75 \mu \mathrm{m}$ in all directions and a recording width of approx. $7.5 \mathrm{~cm}$. The sensor was installed directly in front of the right measurement tyre of the CPX trailer. Due to the high amount of storage usage, section lengths were chosen as approx. $500 \mathrm{~m}$. At this length, it is assumed that a representative part of the section is captured by the measurement.

\subsection{Principal component analysis of CPX data}

As different generation mechanisms of tyre/road noise take effect in different acoustic frequency areas, a frequency-dependent approach for identifying the texture influence on the noise emission must be chosen. As CPX levels are typically measured as overall or one-third octave band levels, a third-band resolution may be selected. Thereby, models for the CPX frequency span of 315$5000 \mathrm{~Hz}$ would be determined. In order to reduce the amount of models, and thus gaining clarity, the one-third octave band CPX dataset at first is analysed with regard to its interrelations. This is accomplished via a principal component analysis (PCA) of the one-third octave band levels of the whole dataset. As can be seen from its visualization of the loading strength of the first three principal components in Figure 1 as well as the described variance by principal component (Fig. 2), the main information is already included in the first three principal components.

A second approach to identify frequency areas exhibiting a comparable behaviour is by calculating their Pearson correlation matrix (Fig. 3). Both methodologies result in 


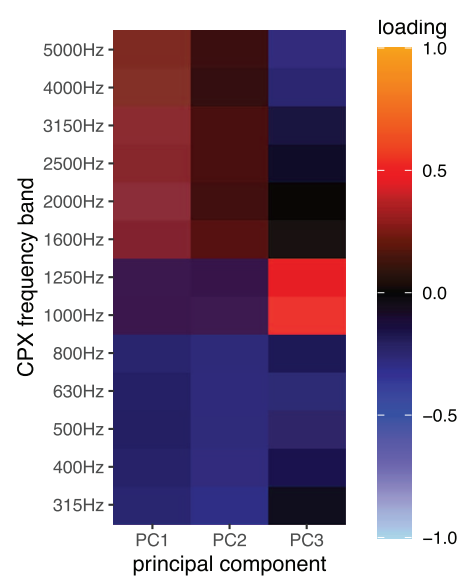

Figure 1. Loading vectors of the first three principal components of the CPX one-third octave band PCA, the loading strength is color-encoded.

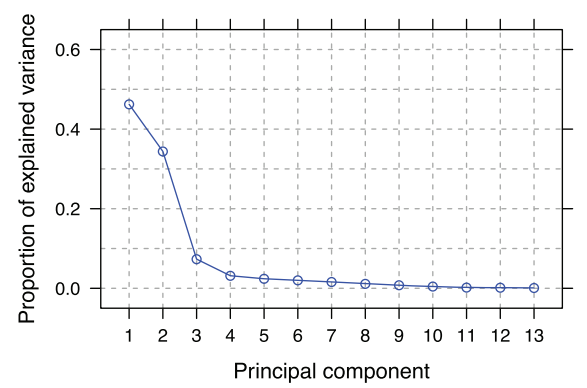

Figure 2. Importance of the principal components of the CPX frequency bands.

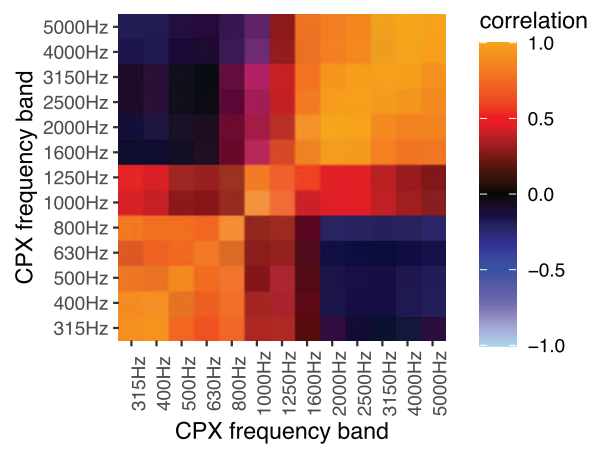

Figure 3. Pearson autocorrelation matrix of the CPX one-third octave bands.

three adjoined frequency areas. Thus, statistical modelling will be performed for these three frequency spans (see Tab. 1). In order to aggregate the one-third octave bands, energetic summation within the groups is performed. Thereby, the model inputs within the three frequency areas are dominated by the louder bands under the assumption of comparable variation within each frequency band.

It needs to be kept in mind that this grouping of the one-third octave bands is only valid for the specific dataset used. Especially other road surfaces, where noise generation
Table 1. Center frequencies of the CPX one-third octave band limits for the three model frequency ranges.

\begin{tabular}{lcc}
\hline Indication & Lower frequency & Upper frequency \\
\hline Low & $315 \mathrm{~Hz}$ & $800 \mathrm{~Hz}$ \\
Mid & $1000 \mathrm{~Hz}$ & $1250 \mathrm{~Hz}$ \\
High & $1600 \mathrm{~Hz}$ & $5000 \mathrm{~Hz}$ \\
\hline
\end{tabular}

mechanisms may be excited with different weights, may exhibit different frequency groups. Also, measurements at different measurement speeds may result in shifts of frequency components to different one-third octave bands.

\section{$2.23 \mathrm{D}$ texture}

The 3D texture scans that are used in this analysis were obtained with the 3D texture measurement system that is described in [27]. This system is a line-scan stereo system which captures the texture as well as the depth of a scanned object and is mounted on the CPX trailer (Fig. 4). As the sensor is installed directly in front of the CPX measurement tyre, it is ensured that the surface texture measured can be interpreted as the input function of the tyre. Although the sensor might cause additional acoustic reflections towards the CPX microphones, the transfer path can be seen as constant. Influences from the sensor therefore will not alter the correlations shown in the next sections.

The system consists of two verged line-scan cameras and an illumination module. The optical axes of the setup are verged to obtain a larger overlapping region. Given the two images from these cameras that were taken from the slightly shifted viewpoints (resp. angles), a 3D model can be estimated by determining pixel correspondences between the images [27, 28] (See Fig. 5).

Compared to conventional area cameras, line-scan cameras are able to acquire objects continuously and at high-speeds - two indispensable properties when scanning road surfaces while driving. Using a high optical resolution of approx. $75 \mu \mathrm{m}$, the max. acquisition speed of the 3D texture scanner is limited to $60 \mathrm{~km} / \mathrm{h}$. The setup covers a stripe of $7.5 \mathrm{~cm}$ in front of the CPX tyre, which corresponds to a line length of 1024 pixels.

\subsubsection{D texture data processing}

When trying to characterise the road surface with 3D texture measurements, multiple data processing steps have to be performed in order to avoid calculation uncertainties and information loss of the sought-after texture parameters. First, the size of the single surface texture patch needs to be considered. This should, depending on the purpose of the further analysis, cover multiple wavelengths of the longest influencing spatial wavelength. In most cases relating to effects based on the tyre/road contact zone (as tyre/road noise for smooth road surfaces in good maintenance condition, as used in the present dataset), this will be the maximum aggregate size of the road surface. Here, a texture patch size of approx. $75 \times 75 \mathrm{~mm}$ was chosen 


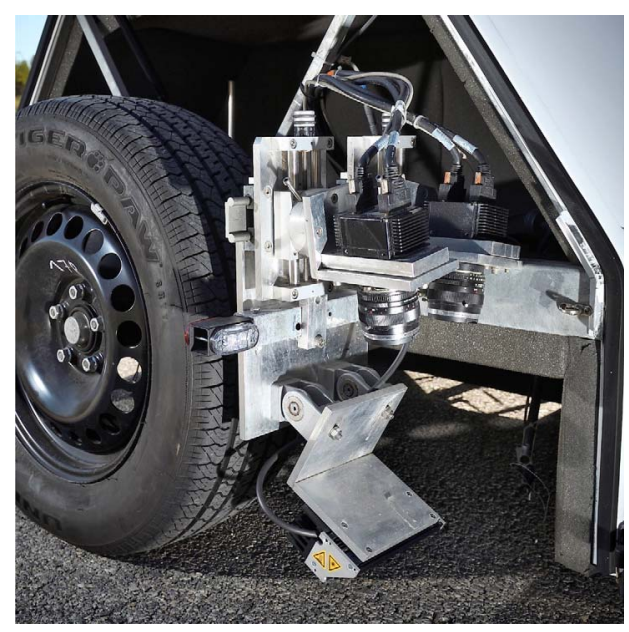

Figure 4. Photo of the texture scanner mounted in front of the CPX measurement tyre (AIT/Zinner).

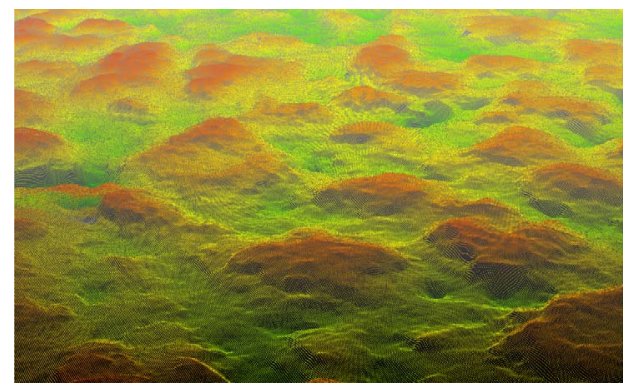

Figure 5. Example of a 3D texture measurement: 3D view of single aggregates (the height is color-encoded).

covering 6 wavelengths of a typical road surface with $11 \mathrm{~mm}$ maximum aggregate size.

Secondly, as the measured road surface may be tilted relatively to the sensor due to side slopes, in order to prevent errors in gradient-related calculations, some kind of surface levelling has to be applied to the measured surface. Here, multiple possibilities exist, e.g., a line-wise levelling in longitudinal or lateral direction, levelling via fitting of a horizontal plane, etc. In the current dataset, this levelling was performed by subtracting a gaussian blur filter working on the height dimension of the texture from the original texture. This is equivalent to the subtraction of a low-pass filter, where the cutoff frequency is determined by the standard deviation of the Gaussian function, i.e., the geometric area of the filter. The cutoff frequency has to be chosen with care, as if the blurring area of the gaussian filter is chosen too low, information in the size range of e.g., the surface aggregates may be lost, if chosen too high, the sought after levelling may not be applied properly.

In a final preprocessing step, as further parameter calculations were performed on separate 3D texture patches, effects of the patch boundaries need to be treated. This was accomplished by a standard windowing approach, where a flat-top hanning window was applied on each texture patch.
With these normalized texture patches, a multitude of different texture parameters may be calculated. Most of these parameters are dependent on the patch area, or even on meta-parameters as described in the following example:

One basic parameter used in the following analyses is the number of local maxima found in the texture patch. It can easily be comprehended that this will be strongly dependent on the patch size, as well as the windowing shape and size described in the previous paragraphs. Additionally to this, the measured surface texture, dependent on the spatial sampling rate of the texture sensor, may exhibit a different microscopic roughness. Therefore, in order to only estimate the most prominent peaks of the texture, a lowpass filter needs to be applied to the texture removing unwanted high-frequency roughnesses. Here, the filter parameters may also be chosen dependening on the subsequent data usage. Therefore, the calibration of these meta-parameters will strongly influence the usefulness of the texture parameter.

In the field of road surface characterization and tyre/ road contact modelling, as automated measurements of 3D texture over long distances are relatively new, no definite normative guidelines for the calculation of texture parameters exist. This is even more complicated, as different surface properties (as e.g., tyre/road noise and skid resistance) may be affected by comparable surface parameters of different spatial extent. In addition, surface characteristics may be only dependent on a part of the texture: in the field of tyre/road noise, the generation mechanism of tyre vibrations is only dependent on the road texture in contact with the tyre, whereas air pumping mechanisms may be primarily dependent on the cavity amount and structure below the tyre/road contact zone.

In the dataset used for the analyses, uncertainties relating to the preprocessing and parameter calculation steps should be of minor influence. This is due to the fact that all measurements were performed with the same measurement system and all calculations were performed within the same software framework. Nevertheless, when comparing the numerical values of the road surface parameters, the origin and processing of external datasets must be kept in mind.

\subsubsection{Principal component analysis}

In order to describe the road surface, different texture parameters were calculated. It needs to be kept in mind that the texture parameters are partly influenced by hyperparameter finetuning, and also the focus of the article is not laid on their exact definitions. Therefore a precise specification of the texture parameter calculations is not given here. As these parameters are the basis for the modelling in Section 3, one main demand is for them to be meaningful and easily interpretable. Following, a short description of the mainly used surface parameters and their abbreviation used in the article is given:

- nmax: number of local maxima per square meter, as calculated from one texture patch. In the scope of this article, the pre-processing steps are adjusted so that 
this roughly refers to the number of stone aggregates within the patch.

- hmax: the median height of the texture at the position of the nmax, relative to the mean height of the texture patch. In combination with hmin this gives an estimate of the visible texture depth.

- dmax: the range of the hmax, calculated from 5 to 95\%-percentiles in order to be robust against outliers. This parameter describes the height variability of the prominent peaks of the texture.

- nmin: the number of local minima per square meter, as calculated from one texture patch (see nmax).

- hmin: the median height (depth) of the texture at the position of the nmin, relative to the mean height of the texture patch (see hmax).

- dmin: the range of the hmin, calculated from 5 to 95\%-percentiles in order to be robust against outliers.

- mpd: mean profile depth, comparable, but not equivalent parameter to the calculation procedure in [29]. The texture patch is split in two halves, and the average of the height (compared to the average level) of the most prominent peaks is calculated. In comparison to the MPD, the spatial wavelength influencing this parameter is considerably smaller.

- $g$ : gestaltfactor, comparable, but not equivalent parameter to the $2 \mathrm{D}$ calculation procedure [30]. The calculation of this parameter equivalent to the gestaltfactor, but the 3D texture patch is used for the height distribution.

- nneigh: mean minimum horizontal distance of each local maximum to its nearest neighbour in the texture patch (nearest-neighbour-distance).

- mas: maximum aggregate size, as estimated from the nmax and a cubic closest packing sphere model.

- $\operatorname{grad}_{x}$ : gradient of the texture patch in longitudinal direction. Calculated as integral of the absolute point height difference in longitudinal direction.

- grad : gradient of the texture patch in lateral direction. Calculated as integral of the absolute point height difference in lateral direction.

- grad: overall gradient of the texture patch.

It can be assumed that interdependencies and correlations exist inbetween these texture parameters. Therefore, as in Section 2.1 for the CPX data, a principal component analysis of the texture parameters was performed. The importance of the first principal components is shown in Figure 6. Again, the main information gained from the texture measurements can be summarized in the first two principal components. In Figure 7, the loading vectors of the first three principal components are depicted. When interpreting the contributing parameters to the components, a separation of gradient-related texture parameters in the first and surface structure-related parameters in the second principal component can be assumed. The first principal component can be seen as description of the depth structure of the surface. Height- and depth-information (hmin, hmax, mpd, dmin, dmax) as well as - via the gradients and the gestaltfactor - the type of depth structure is included, whereas this segregation is represented in the third principal component. The parameters of the second

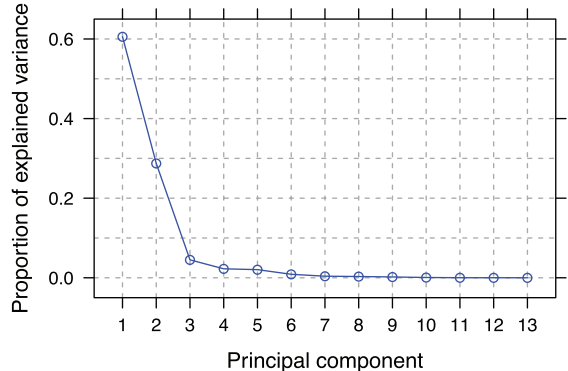

Figure 6. Importance of the principal components of the texture parameters.

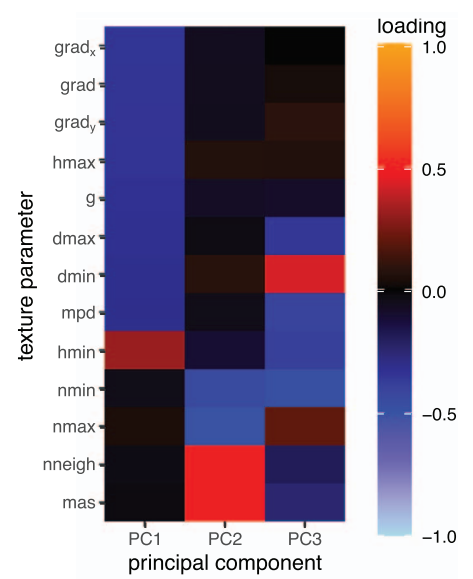

Figure 7. Loading vectors of the first three principal components of the PCA, the loading strength is color-encoded.

principal component (nmin, nmax, nneigh, mas) describe the aggregate structure with regard to its packing density viz. maximum aggregate size.

Again, this categorization should be only seen as valid within the current dataset consisting of SMA surfaces. Especially for road surfaces with diverging grading curves, as asphalt concrete or exposed-aggregate cement-concrete surfaces (or even more blatant, anisotropic surfaces as diamond-grinding textures), diverging categorizations may be found. Nevertheless, the principal component analysis substantiates that the basic surface structure can be described with two interpretable groups of texture parameters. The calculation of additional texture parameters may be sought in the future, based on specific problem definitions, although it should be verified that thereby additional information is added to the dataset.

\section{Modelling approach}

In order to test the prediction capabilities of 3D texture measurements on tyre/road noise, two approaches were pursued.

First, a basic linear modelling of the low, mid, and high frequency range as determined in Section 2.1 was performed using the principal components of the texture parameters 
(see Sect. 3.1). This model is supposed to give a first glimpse on the potential of identifying correlations between texture and tyre/road noise, without stating physical interpretations of the reasons for these correlations. Also, the linearity confines the model to a narrow range of texture - tyre/road noise interrelations.

Second, a more elaborate modelling approach based on the individual texture parameters utilizing random forest regression models was chosen (Sect. 3.2). Although still operating on the aggregated frequency ranges, nonlinearities due to the random forest regression approach will be allowed.

For both approaches, the texture parameters introduced in Section 2.2.2 will be used. Although also texture data described by the (complex) 2D fourier spectra of the road surface (by e.g., aggregation of third-octave bands) or constant-Q transforms could be used, intuitive interpretability, which was tried to preserve by the parameters, might suffer thereby.

\subsection{Linear modelling via principal components}

As first, direct approach to model the interrelations between road texture and CPX levels, a linear univariate modelling approach was performed according to,

$$
\hat{L}_{\mathrm{CPX}, f}=\beta_{0}+\beta_{1} \cdot \mathrm{PC},
$$

where $\hat{L}_{\mathrm{CPX}, f}$ denotes the CPX level of the frequency range $f, \beta$ the coefficients and $\mathrm{PC}$ the principal component used for the model. Based on the low, mid and high frequency CPX aggregates and the first three principal components of the 3D texture, a scatter plot matrix is shown in Figure 8. In the lower triangle, the individual scatter plots of the different dataset components are shown, whereby the different SMA types (SMA S1 and SMA S3) are color-encoded. Supplementary, the coefficients of determination of a linear modelling of the dataset components are shown in the upper triangle (where the font size also resembles the value). Finally, the kernel density estimate of the values is depicted in the scatter plot matrix diagonal. Not surprisingly, the frequency-aggregated CPX levels show no or low correlations, as could be expected from the pearson correlation matrix in Figure 3. The correlations between the principal components, as a direct implication of the PCA, are zero.

When considering the interrelations of the CPX levels and the principal components, noteworthy correlations appear for all three frequency ranges: For low-frequency CPX components, a coefficient of determination of 0.70 can be found with the first principal component. The mid-frequency CPX aggregate, which strongly influences the overall CPX level, even exhibits a coefficient of determination of 0.78 . The high-frequency CPX level correlation shows a value of 0.53 . This is insofar interesting, as no information regarding the void content of the road sections is included in the principal components, whereby an influence of this void content may be expected for high-frequency air pumping generation mechanisms. The only parameters possibly interrelating with the void content may be hmax

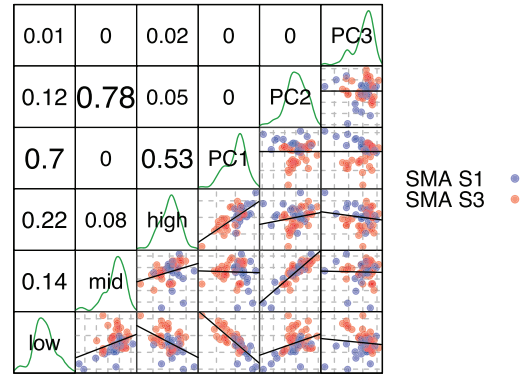

Figure 8. Correlations of low-, mid- and high-frequency CPX levels with the 3D texture principal components. The lower triangle depicts the individual scatter plots, the upper triangle the coefficient of determination of the respective linear regression. The parameter names in the diagonal denote the variables on the axes of the matrix elements, e.g. the lowest row depicts the individual scatter plots of mid, high, and PC1 to PC3 variables on the abscissa vs. the low-frequency component on the ordinate. In addition, the diagonal elements also show the variables' kernel density estimate.

Table 2. Performance parameters for the linear model based on texture PCA data: mean absolute deviation (MAD), root mean squared error (RMSE) and coefficient of determination $\left(R^{2}\right)$ for the three frequency ranges.

\begin{tabular}{lcccc}
\hline Frequency range & MAD $[\mathrm{dB}]$ & RMSE $[\mathrm{dB}]$ & $R^{2}$ & Predictor \\
\hline Low & 0.56 & 0.70 & 0.70 & PC1 \\
Mid & 0.33 & 0.43 & 0.78 & PC2 \\
High & 0.63 & 0.81 & 0.53 & PC1 \\
\hline
\end{tabular}

and $h$ min, which together describe the (visible) depth information of the road surface. Actually, they are loading in the first principal component, which mainly contributes to the high-frequency correlation. An overview of the performance of the linear models is given in Table 2.

Moreover, interesting interrelations between the highvalued correlations exist: Where the mid-frequency range is solely described by the second principal component (which to some extent resembles the packing density of the road surface), both low- and high-frequency ranges of the dataset can be estimated via the first principal component. Here, on the one hand, the gradients of the interrelations show opposing behaviour. On the other hand, the two SMA types within the dataset interchange: where SMA S1 surfaces exhibit lower low-frequency CPX components, for the high frequency range, SMA S3 surfaces perform slightly better.

Finally, when calculating multivariate models for the low, mid, and high frequency range based on the three principal components according to,

$$
\begin{array}{r}
\hat{L}_{\mathrm{CPX}, f}=\beta_{0}+\beta_{1} \mathrm{PC}_{1}+\beta_{2} \mathrm{PC}_{2}+\beta_{3} \mathrm{PC}_{3}+\beta_{1,2} \mathrm{PC}_{1} \mathrm{PC}_{2} \\
+\beta_{1,3} \mathrm{PC}_{1} \mathrm{PC}_{3}+\beta_{2,3} \mathrm{PC}_{2} \mathrm{PC}_{3}+\beta_{1,2,3} \mathrm{PC}_{1} \mathrm{PC}_{2} \mathrm{PC}_{3},
\end{array}
$$

the low- and high-frequency models yield significant improvements compared to the univariate linear model. 


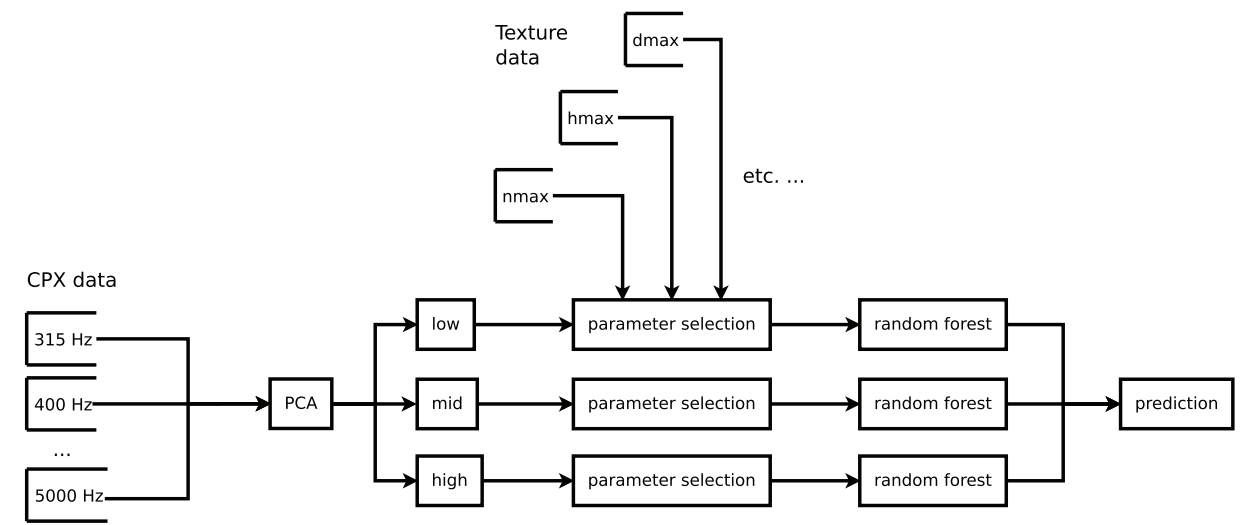

Figure 9. Block diagram of the multivariate modelling approach.

For the low-frequency range, the coefficient of determination may be enhanced to 0.88 with a mean absolute deviation of $0.36 \mathrm{~dB}$; for the high-frequency model, a coefficient of determination of 0.65 and a mean absolute deviation of $0.30 \mathrm{~dB}$ are obtained. Nevertheless, these models should be treated with care, as interaction terms were allowed resulting in a high number of model input parameters and risking model overfitting. Therefore, this model approach will not be followed further on in this article.

As final step in the linear modelling process, a combined model based on the three univariate frequency submodels can be calculated by energetic summation of the submodel predictions:

$$
\hat{L}_{\mathrm{CPX}}=10 \cdot \log _{10} \sum_{f} 10^{0.1 \cdot \hat{L}_{\mathrm{CPX}, f}} .
$$

Its results, performance and comparison with the random forest models introduced in Section 3.2 will be discussed in Section 4.

\subsection{Frequency-dependent texture parameter model}

For modelling the influence of the individual road surface texture parameters on tyre/road noise, a four-stage modelling approach was chosen (see Fig. 9). First, the CPX data was split into three independent, energy-summed datasets based on the findings in Section 2.1. This separation, in contrast to a direct modelling of the overall CPX level, is necessary in order to consider multiple tyre/road noise generation mechanisms.

Second, for each dataset, the choice of the influence parameters was performed according to the following steps:

1. The dataset was split into training and test data $(75 \%$ training dataset size).

2. For each two-parameter-combination (to avoid overfitting) of texture parameters $\left(P_{i}, P_{j}\right)$, a multivariate model (including the interaction term) was built:

$$
\hat{L}_{\mathrm{CPX}, f}^{i, j}=\beta_{0}^{i, j}+\beta_{i}^{i, j} P_{i}+\beta_{j}^{i, j} P_{j}+\beta_{i, j}^{i, j} P_{i} P_{j} .
$$

3. The model validity was checked by calculating the coefficient of determination based on the test dataset.
4. The steps $1-3$ were repeated 1000 times, based on different training/test random samples of the dataset.

5. For each cycle, the best-performing two-parameter combination $\left(P_{i}, P_{j}\right)$ was chosen.

6. Based on the frequency distribution of this cross-validated grid search, the two-parameter combination with most occurences as best-performing combination for each frequency range was chosen for further modelling.

Third, based on the parameter choice, in order to allow for slight nonlinearities, for each frequency range a randomforest regression model was calculated:

$$
\hat{L}_{\mathrm{CPX}, f}=\frac{1}{B} \sum_{b=1}^{B} \hat{L}_{\mathrm{CPX}, f}^{*}
$$

with $B$ denoting the number of cross-validated grid search runs,

$$
\hat{L}_{\mathrm{CPX}, f}^{*}=\sum_{m=1}^{M} c_{m} \cdot 1_{\left(P \in R_{m}\right)},
$$

the single regression tree, $c_{m}$ the coefficients, $1_{\left(P \in R_{m}\right)}$ a unity matrix describing the splits of the regression tree, $P$ the two texture parameters and $R_{m}$ the partitions of the feature space [31]. Here, due to the linear modelling during the parameter choice, no strong nonlinearities are to be expected. This focus on linearity, on the one hand, was chosen in order to avoid overfitting (which although was not expected within the parameters' data range). On the other hand, interpretability of the model parameter choice was sought, which is easier established for steadily rising/falling model relationships.

Finally, the outputs of the three submodels were combined by energetic summation to predict the overall CPX level as in (3). Here, the overall model performance can be calculated by a reevaluation of the assembled submodels. A separation in test/training datasets is not meaningful at this stage, as the model optimization procedure was performed for the frequency-dependent submodels, where the three models were trained independently from each other and the calculation of the overall value. 


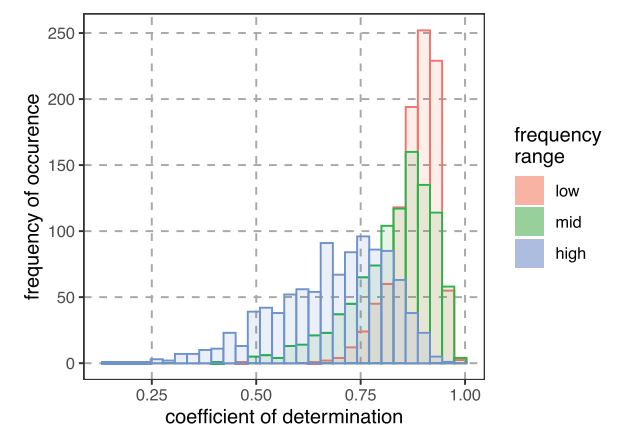

Figure 10. Distribution of the best two-parameter model coefficients of determination resulting from the 1000 crossvalidated grid search runs.

\subsubsection{Parameter selection}

As described above, the parameter selection for the three CPX frequency ranges was performed by testing different multivariate linear two-parameter models for multiple training/test data sets. For each training/test dataset slice, the best model parameter selection was kept. The distribution of the associated coefficients of determination is depicted in Figure 10 for the three frequency CPX levels. In a predominant part of the model test runs, a satisfactory value for the coefficient of determination is reached. Especially for the low-frequency range, the cross-validated grid search results in a maximum of the distribution of above 0.8 , meaning that for each dataset slice, a highly performant two-parameter model can be found.

The occurence of the best parameter combinations for low frequencies is shown in Figure 11. In approx. 175 of 1000 cases, the best parameter choice consists of the number of local maxima per square meter nmax in combination with the texture gradient in driving direction $\operatorname{grad}_{x}$. This parameter selection is followed by nmax combined with $\mathrm{grad}_{y}$; as $\mathrm{grad}_{x}$ and $\mathrm{grad}_{y}$ are highly correlated texture parameters for isotrope road surfaces, the combination of nmax and a directional $\operatorname{grad}_{x / y}$ can be considered as best model choice in over $25 \%$ of the cross-validated grid search runs. Not surprisingly, the first seven parameter combinations consist of texture parameters assigned to different loadings in the principal component analysis of Section 2.2.2. As first single-PCA combination, the parameters of hmin and mpd both load on the first principal component, which was found to be the best-correlated principal component to the low-frequency CPX levels in the linear modelling approach of Section 3.1.

Alike, the feature selection process was performed for the mid-frequency CPX levels. Here, also with comparable levels of the coefficient of determination distribution, as most frequent combination the nmax and the nneigh parameters are responsible for more than $25 \%$ of the best parameter combinations. This is insofar interesting, as both nmax and nneigh mainly load the second principal component of the 3D texture PCA. As second-best combination, the nneigh are identified with the dmax parameter in approx. $15 \%$ of the cross-validated grid search runs. nneigh

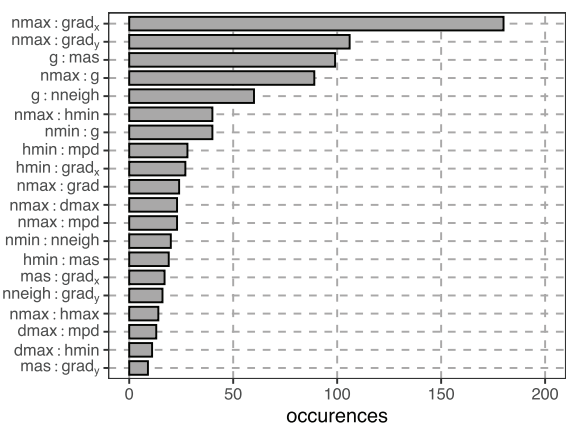

Figure 11. Histogram of the occurences of the best choices of the low frequency two-parameter model feature selection process.

can even be found in the first five parameter combinations and is thereby selected in approx. $70 \%$ of the crossvalidated grid search combinations as relevant parameter. This may lead to the assumption, that not only the number of local maxima per square meter, but even more their spatial distribution has a strong influence on mid-frequency CPX levels.

For the high-frequency CPX levels, the distribution of the coefficients of determination results in a slightly poorer model accuracy estimation. The maximum of the distribution is broadened in comparison to the low- and midfrequency calculations, and although the maximum is still at approx. 0.75, a relevant amount of the cross-validated grid search runs results in a best coefficient of determination of below 0.5 . As mainly selected texture parameter, hmax, describing the visible depth structure of the road surface, appears in the first four linear model selections accounting for more than $50 \%$ of the cross-validated grid search runs. The best parameter choice is identified as hmax in combination with nmax, followed by the combination of hmax and nneigh.

\subsubsection{Random forest regression}

Based on the parameter selection in the previous section, three independent random forest regression models were built [32]. The results of the models are shown in Figures 12-14. The model evaluations are color-encoded in the plots according to the predictor values. Additionally, the density of the input dataset is depicted via the dashed contour lines. Thereby, strongly supported areas of the modelling can be distinguished from areas where extrapolation occurs. The results of the performance measures is given in Tables 3 and 4 .

For the low-frequency CPX range, the model is supported for a broad parameter range both for the nmax and $\operatorname{grad}_{x}$. The approximately round shape of the input density distribution is a direct result of non-correlating modelling parameters loading on different principal components in Section 2.2.2. The model accuracy can be described by the root mean squared error of $0.7 \mathrm{~dB}$. This is no further improvement compared to the linear modelling using the principal components in Section 3.1 (which was not to be 


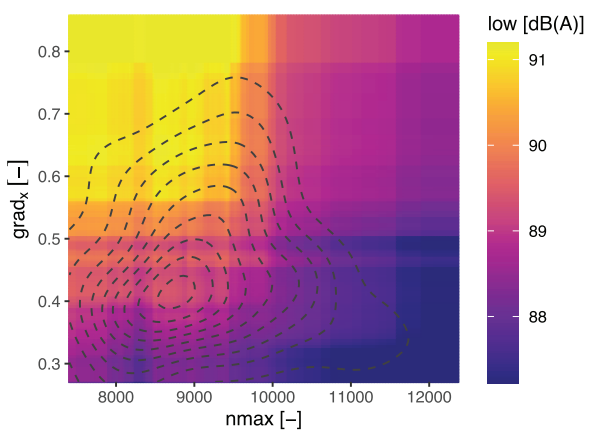

Figure 12. Visualization of the low frequency random forest predictions; the estimated CPX levels are color-coded, the dashed contour lines denote the kernel density estimates of the input data, thus marking the areas of model inter- and extrapolation.

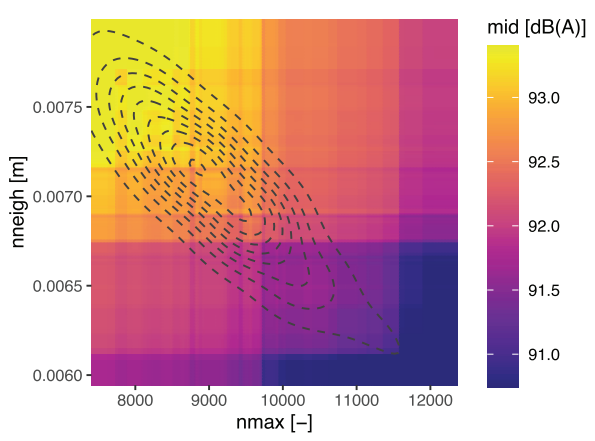

Figure 13. Visualization of the mid frequency random forest predictions; the estimated CPX levels are color-coded, the dashed contour lines denote the kernel density estimates of the input data, thus marking the areas of model inter- and extrapolation.

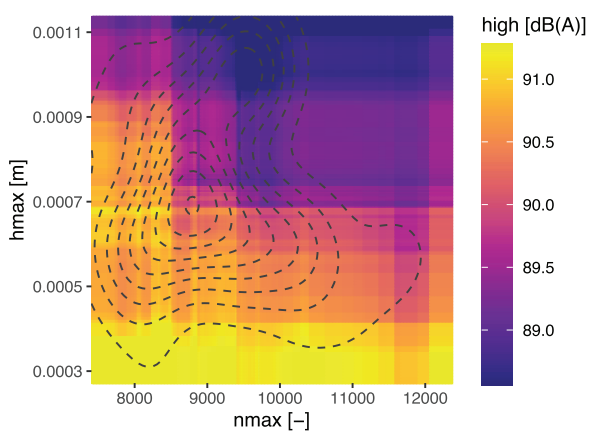

Figure 14. Visualization of the high frequency random forest predictions; the estimated CPX levels are color-coded, the dashed contour lines denote the kernel density estimates of the input data, thus marking the areas of model inter- and extrapolation.

expected due to the parameter selection based on linear models), but increases interpretability: high low-frequency CPX levels are mainly found for high values of $\operatorname{grad}_{x}$ and low amounts of nmax. The extrapolation of the model suggests that by increasing the nmax towards the highest
Table 3. Out-of-bag performance parameters for the random forest model based on texture parameters: mean absolute deviation (MAD), root mean squared error (RMSE) and coefficient of determination $\left(R^{2}\right)$ for the three frequency ranges.

\begin{tabular}{lccc}
\hline Frequency range & MAD (oob) $[\mathrm{dB}]$ & RMSE $(\mathrm{oob})[\mathrm{dB}]$ & $R^{2}$ \\
\hline Low & 0.54 & 0.73 & 0.69 \\
Mid & 0.38 & 0.53 & 0.66 \\
High & 0.67 & 0.90 & 0.42 \\
\hline
\end{tabular}

Table 4. Reevaluation performance parameters for the full random forest model based on texture parameters: mean absolute deviation (MAD), root mean squared error (RMSE) and coefficient of determination $\left(R^{2}\right)$ for the three frequency ranges.

\begin{tabular}{lccc}
\hline Frequency range & MAD (full) $[\mathrm{dB}]$ & RMSE (full) $[\mathrm{dB}]$ & $R^{2}$ \\
\hline Low & 0.25 & 0.33 & 0.95 \\
Mid & 0.18 & 0.24 & 0.93 \\
High & 0.31 & 0.41 & 0.91 \\
\hline
\end{tabular}

values found in the measured input dataset and simultaneously keeping the $\operatorname{grad}_{x}$ below a threshold of 0.5 , lowfrequency CPX levels can be reduced by approx. $2 \mathrm{~dB}$ from the mean value found in the dataset and approx. $4 \mathrm{~dB}$ from the highest occuring levels. Here, it has to be kept in mind that the model input dataset consists of SMA surfaces of the same categorical maximum aggregate size. Therefore, only slight variations within the grading curve, as well as ageing effects restructuring the texture/tyre contact area, result in significantly higher low-frequency CPX levels.

In the mid-frequency CPX range (Fig. 13), the input parameter density distribution of nmax and nneigh shows a deviating behaviour compared to low frequencies: the strongly directional density distribution is a result of the highly correlated input parameters, both loading on the second principal 3D texture component. High amounts of nmax are mainly correlated to low values of nneigh, which for anisotropic road surface textures is to be expected. Only for inhomogeneous spatial distributions of the stone aggregates, deviations from this direct correlation may be expected. With an out-of-bag root mean squared error of approx. $0.5 \mathrm{~dB}$, the model accuracy can be estimated as useful for the present objectives. The interpolated CPX level range for mid frequencies is slightly lower than for the low frequency range. Low amounts of nmax result in a CPX level reduction of approx. $2 \mathrm{~dB}$ from the highest occurring values, whereas the linearity in the populated dataset, seen via the diagonal in the Figure, combined with the good model accuracy, suggests further potential in reducing CPX levels by increasing the amount of nmax towards the model extrapolation area.

In Figure 14, the visualization of the high-frequency CPX level random forest model is depicted. The modelling parameter density distribution exhibits a wide variety of the nmax and hmax (as both load on different principal components), extrapolation occurs mainly for high amounts 
of $n \max$ and high values of hmax in the graphic. The random forest regression model exhibits an out-of-bag mean squared error of $0.9 \mathrm{~dB}$, indicating it as the frequency model with the poorest benchmark parameter. As suspected from the linear modelling based on the principal components, the main influence factor on the high-frequency CPX levels is the hmax parameter. High values of hmax, representing a large (visible) depth structure in the road surface texture, may lead to low high-frequency CPX levels. The limitation to the visible depth structure here rests upon the measurement principle of the 3D texture, where an optical method is used that is incapable of measuring porous structures of the road surface. This may be a reason for the lowest benchmark parameter of the three frequency-dependent random forest regression models, although the input dataset road surfaces exhibit mainly low void contents.

\section{Combined overall CPX level model}

Based on the frequency-dependent submodels, by energetic summation of the predictions an overall model can be developed. In contrast to the linear modelling approach, were all input datapoints were used for a single model creation, this will be done both for the out-of-bag as well as the in-bag random forest predictions separately. The results of the performance measures are given in Table 5 .

The results of the linear modelling reevaluation are depicted in Figure 15. As the performance measures suggest, a suitable recalculation of the overall levels could be achieved. The maximum prediction error can be calculated to approx. $1.5 \mathrm{~dB}$. Interestingly, the slope of the prediction clearly deviates from the diagonal.

As final step in the random forest model building, the submodel outputs can be combined to calculate predicted overall CPX levels. Reevaluating the model output with the full dataset by calculating a linear model based on the out-of-bag prediction (see Fig. 16), the overall performance of the modelling process can be rated. Although the coefficient of determination of the full model is relatively low with a value of 0.56 , the root mean squared error exhibits a promising value of $0.44 \mathrm{~dB}$. Compared to an estimated repeatability of the CPX measurements of approx. $0.15 \mathrm{~dB}$ according to [18] and an expanded measurement uncertainty of $1.0 \mathrm{~dB}$ according to [10] for a coverage probability of $95 \%$, the modelling approach results in suitable prediction errors. The maximum prediction error is found as the same data point as in the linear model with a value of $1.4 \mathrm{~dB}$. In contrast to the linear model, the slope of the correlation is nearly one. As seen from the calculations in Section 3.2.2, main contributions to these errors originate from the high-frequency range with a root mean squared error of $0.9 \mathrm{~dB}$ (high frequency range), $0.7 \mathrm{~dB}$ (low freq. range) and $0.5 \mathrm{~dB}$ (mid freq. range). These values are of similar size as in the approach based on the principal components, but obtained with the use of only four independent $3 \mathrm{D}$ texture parameters.

As the linear model approach was - due to the univariate approach - calculated directly on the full dataset
Table 5. Reevaluation performance parameters for the modelling approaches: mean absolute deviation (MAD), root mean squared error (RMSE) and coefficient of determination $\left(R^{2}\right)$ for the three frequency ranges.

\begin{tabular}{lccc}
\hline Model approach & MAD [dB] & RMSE [dB] & $R^{2}$ \\
\hline Linear model & 0.33 & 0.43 & 0.64 \\
Random forest (oob) & 0.33 & 0.44 & 0.56 \\
Random forest (reeval) & 0.15 & 0.20 & 0.93 \\
\hline
\end{tabular}

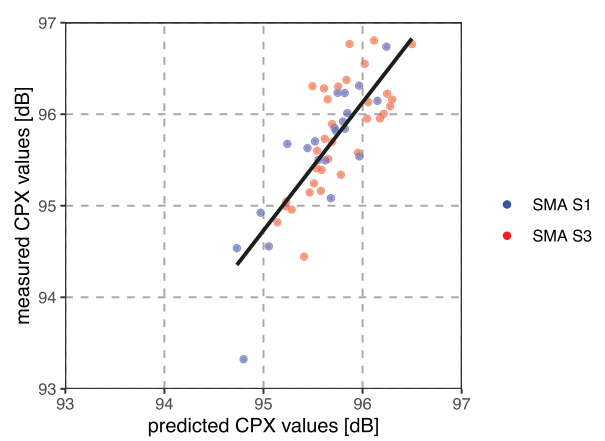

Figure 15. Reevaluation of the combined overall linear principal component CPX model. The black line shows the linear regression model of the prediction.

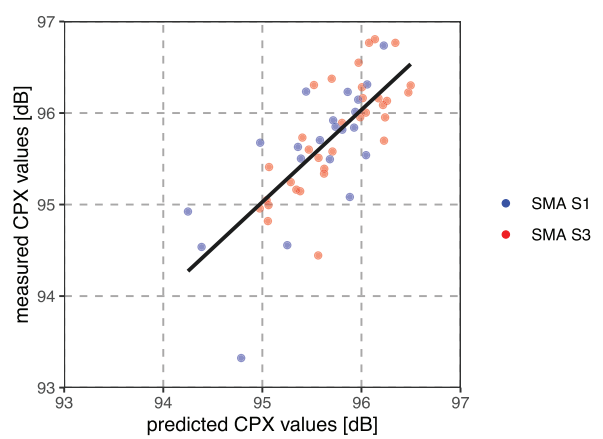

Figure 16. Reevaluation of the combined overall random forest CPX model based on the out-of-bag prediction. The black line shows the linear regression model of the prediction.

instead of the feature selection via cross-validated grid search and bagging for the random forests, a reevaluation of the final random forest models is given in Figure 17. This results in a more comparable inter-model comparison between the linear and random forest approach. Here, we see that the random forest allows for corrections for nonlinearities without losing the nearly diagonal slope in the prediction. The performance measures exhibit a clearly better value as the linear model with a maximum prediction error reduced to approx. $0.6 \mathrm{~dB}$, although for the overall model performance, the benchmark parameters of the out-of-bag predictions should be considered.

Generally speaking, when building statistical models for the purpose of predicting rolling noise levels based on texture parameters, more robust estimations of the model performance will be reached by use of a separate test data 


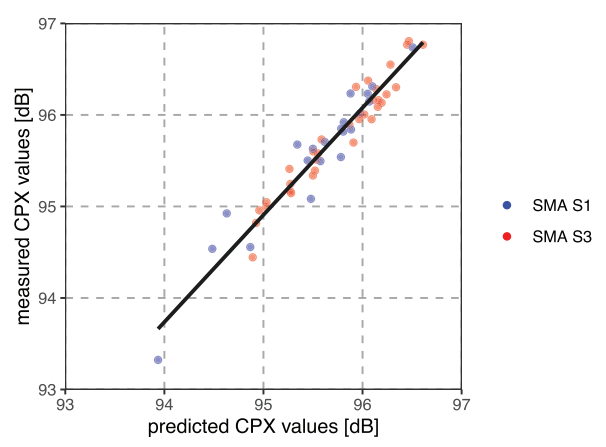

Figure 17. Reevaluation of the combined overall random forest CPX model based on the final model prediction. The black line shows the linear regression model of the prediction.

set. In the work described here, this was not implemented due to two reasons: once, due to the high cost of the data acquisition, only a limited dataset could be gathered. Second, the overall prediction performance was of subordinate interest. As described in Section 5, mainly the contributing texture parameters to the generation of rolling noise were sought to be identified.

\section{Discussion of the model results}

When comparing the two modelling approaches linear modelling via principal components and random forest regression modelling based on direct 3D texture parameters - similar values for benchmarking parameters could be achieved. Nonlinearities were prohibited in the univariate PCA-approach resp. avoided for the random forest regressions in order to maintain easy interpretability.

Where the univariate linear approach suggests strong influences of the depth structure for low-frequency CPX levels, the random forest regression approach reveals both influences of the surface gradient as well as - to a lesser extent within the dataset range - the amount of local maxima nmax. In an attempt to physically interpret these results, road surfaces with low gradients in combination with high amounts of nmax may yield in a more constant contact deformation of the tyre rubber within the tyre/road contact zone, thus leading to lower tyre vibrations. To further substantiate this assumption, calculations of the rubber deformations may be suitable. Subsequently, the texture parameters can be recalculated for the deformed tyre rubber, and thereby only describing the actual contact between the tyre and the road surface.

For the mid-frequency CPX levels, as primary contribution to the models, the aggregate structure is most relevant. The amount of local maxima nmax as well as its packing density via the nearest-neighbour-distance nneigh show strong influence on the CPX levels. This is most interesting with regard to the dataset consisting only of SMA surfaces with a maximum aggregate size of $11 \mathrm{~mm}$. The main reason for the variation within the parameters in the dataset is based on different installation qualities and ageing effects of the road sections. Here, the strongly relevant nmax should be further analysed. In its current form, this parameter directly counts the number of local maxima within the texture patch with the potential of finding multiple local maxima per stone aggregate. A further improvement of the parameter calculation, e.g., a split into the number of aggregates and the dominant rubber deformation locations may help in finding more suitable surface parameters.

When analysing the model qualities for the highfrequency CPX levels, lower rates of the benchmark parameters are found for both approaches. Where the linear modelling of principal components suggests an opposing behaviour of the first principal component with regard to low-frequency emissions, the random forest regression approach points towards a more differentiated view. There, mainly the texture depth, represented via the hmax is found to be influencing the high-frequency CPX levels. Therefore, a combined optimization of the road surface texture for all frequency ranges may be possible. High amounts of local maxima, leading to a constant rubber deformation in the tyre/road contact, with simultaneous deep structures within the road texture, are a viable approach to low-noise road surfaces with low void content.

All of these considerations - although not novel - are insofar interesting, as they are drawn from a dataset with very limited input data ranges. Correlations of single texture parameters within a narrow class of road surfaces are not expected to be a sure success. This substantiates the approach of finding causes of rolling noise emissions by combined measurements of road surface texture and tyre/road noise. Even more, effects of small variations within the road surface texture may be further on analysed. In the dataset presented, reasons for ageing effects within the SMA dataset could be revealed by use of easily interpretable surface texture parameters.

$3 \mathrm{D}$ texture measurements in combination with statistical analyses based on different modelling approaches can be seen as a tool to further interpret the interrelationships between the road surface texture and rolling noise emissions. Physical modellings of the tyre and/or rubber deformations are expected to further improve the models and thereby verify the parameter validity. Thereby, modelling benchmarks can also be used as test of physical models, as by incorporating additional parameters in the statistical modelling process, their validity on tyre/road noise emissions is expected to result in models with higher prediction accuracy.

\section{Summary}

In this article, statistical modelling approaches of tyre/ road noise CPX levels, based on continuous 3D-texture measurements, where introduced. Different approaches, based on the direct texture parameters as well as on a beforehand performed principal component analysis, were presented. It could be shown that, with use of tangible texture parameters, significant correlations to CPX levels were found. Due to the focus on linearity, both approaches presented in this article result in similar model qualities. 
By use of strongly nonlinear models, higher prediction accuracies, on the cost of interpretability of the interrelations, may be found.

The relevance of continuous 3D texture measurements, combined with rolling noise measurements, on the analysis of the tyre/road contact, could be shown. Also, a generalization of the approach on different road parameters, as skid or rolling resistance, seems viable. Further improvements may be expected by incorporating texture parameters based on the calculation of tyre rubber deformations resp. the contact forces in the tyre/road contact zone.

\section{Conflict of interest}

Authors declared no conflict of interests.

\section{Acknowledgments}

The work presented in this article was performed in the scope of the project ADURA, funded by the Austrian Research Promotion Agency (FFG) in the D-A-CH Kooperation Verkehrsinfrastrukturforschung 2017 - DACH 2017 (project number 863065). The authors would like to thank their project partners from PMS Consult, Grolimund + Partner AG, TU Braunschweig and Müller BBM as well as their project advisor Karl Gragger from ASFINAG.

\section{References}

1. European Environment Agency: Environmental noise in Europe, 2020. Publications Office, 2020. https://doi.org/ $10.2800 / 686249$.

2. WHO Regional Office for Europe: Environmental noise guidelines for the European Region. World Health Organization. Regional Office for Europe, 2018.

3. WHO Regional Office for Europe: Burden of disease from environmental noise: Quantification of healthy life years lost in Europe. World Health Organization. Regional Office for Europe, 2011.

4. Conference of European Directors of Roads (CEDR): CEDR Technical Report 2017-02 State of the art in managing road traffic noise: Noise barriers. CEDR Secretariat, 2017.

5. U. Sandberg, J. Ejsmont: Tyre/road noise reference book. INFORMEX, Sweden, 2002.

6. UNECE: Regulation No 117 of the Economic Commission for Europe of the United Nations (UNECE) - Uniform provisions concerning the approval of tyres with regard to rolling sound emissions and/or to adhesion on wet surfaces and/or to rolling resistance. 2016

7. International Organization for Standardization, ISO 10844:2014: Acoustics - Specification of test tracks for measuring noise emitted by road vehicles and their tyres. Geneva, 2014.

8. T. Berge, U. Sandberg: Five years of eu tyre labelling: Success or failure?, in Proceedings of Internoise. 2017.

9. International Organization for Standardization, ISO 118191:1997: Acoustics - Measurement of the influence of road surfaces on traffic noise - Part 1: Statistical Pass-By method. Geneva, 1997.
10. International Organization for Standardization, ISO 11819 2:2017: Acoustics - Method for measuring the influence of road surfaces on traffic noise - Part 2: Close-proximity method. Geneva, 2017.

11. J. Cesbron, P. Klein: Correlation between tyre/road noise levels measured by the coast-by and the close-proximity methods. Applied Acoustics 126 (2017) 36-46. https://doi. org/10.1016/j.apacoust.2017.05.005.

12. ASTM International - F09 Committee, ASTM F2493 - 14: Standard Specification for P225/60R16 97S Radial Standard Reference Test Tire. West Conshohocken, Pennsylvania, USA, 2006.

13. E. Bühlmann, G. van Blokland: Temperature effects on tyre/ road-noise - A review of empirical research, in Proceedings of Forum Acusticum, 2014.

14. P. Mioduszewski, S. Taryma, R. Wozniak: Temperature influence on tyre/road noise of selected tyres, in Proceedings of Internoise, 2014.

15. E. Bühlmann, U. Sandberg, P. Mioduszewski: Speed dependency of temperature effects on road traffic noise, in Proceedings of Internoise, 2015.

16. U. Sandberg: Standardized corrections for temperature influence on tire/road noise, in Proceedings of Internoise, 2015.

17. U. Sandberg, E. Bühlmann, M. Conter, P. Mioduszewski, R. Wehr: Improving the CPX method by specifying reference tyres and including corrections for rubber hardness and temperature, in Proceedings of Internoise, 2016.

18. R. Wehr, A. Fuchs, C. Aichinger: A combined approach for correcting tyre hardness and temperature influence on tyre/ road noise. Applied Acoustics 134 (2018) 110-118. https://doi. org/10.1016/j.apacoust.2018.01.004.

19. F. Reinink, G. van Blokland, F. de Roo, G. Derksen: Comparison of CPX systems with Round Robin Test, in Proceedings of Internoise, 2012.

20. P. Peeters, F. Reinink, W. van Vliet: Close proximity (cpx) round robin test 2017, in Proceedings of Euronoise, 2018.

21. T. Vieira, U. Sandberg: Close proximity (CPX) round robin test: Comparison of results from four different CPX trailers measuring noise properties of 10 swedish road surfaces, in Proceedings of Internoise, 2019.

22. F. Wullens, W. Kropp: A three-dimensional contact model for tyre/road interaction in rolling conditions. Acta Acustica United with Acustica 90 (2004) 702-711.

23. P. Andersson, W. Kropp: Time domain contact model for tyre/road interaction including nonlinear contact stiffness due to small-scale roughness. Journal of Sound and Vibration 318, 1 (2008) 296-312. https://doi.org/10.1016/j.jsv.2008. 04.013.

24. J. Winroth, W. Kropp, C. Hoever, P. Höstmad: Contact stiffness considerations when simulating tyre/road noise. Journal of Sound and Vibration 409 (2017) 274-286. https://doi.org/10.1016/j.jsv.2017.07.044.

25. W. Kropp, P. Sabiniarz, H. Brick, T. Beckenbauer: On the sound radiation of a rolling tyre. Journal of Sound and Vibration 331, 8 (2012) 1789-1805. https://doi.org/10.1016/ j.jsv.2011.11.031.

26. G. Dubois, J. Cesbron, H. Yin, F. Anfosso-Lédée, D. Duhamel: Statistical estimation of low frequency tyre/road noise from numerical contact forces. Applied Acoustics 74, 9 (2013) 1085-1093. https://doi.org/10.1016/j.apacoust.2013. 03.011 .

27. K. Valentín, R. Huber-Mörk, S. Stolc: Binary descriptorbased dense line-scan stereo matching. Journal of Electronic Imaging 26, 1 (2017) 1-12. https://doi.org/10.1117/1.JEI. 26.1.013004

28. R. Szeliski: Computer Vision. Springer, London, 2011. https://doi.org/10.1007/978-1-84882-935-0. 
29. International Organization for Standardization, ISO 134731:2019: of pavement texture by use of surface profiles Part 1: Determination of mean profile depth. Geneva, 2019.

30. T. Beckenbauer, P. Spiegler, G. van Blokland: Einfluss der Fahrbahntextur auf das Reifen-Fahrbahn-Geräusch. Bundesministerium für Verkehr, Bau- und Wohnungswesen und Forschungsgesellschaft für Strassen- und Verkehrswesen FGSV, Heft 847, Bonn (Germany), 2002.

31. G. James, D. Witten, T. Hastie, R. Tibshirani: An Introduction to Statistical Learning. Springer, New York, 2013. https://doi.org/10.1007/978-1-4614-7138-7.

32. A. Liaw, M. Wiener: Classification and regression by randomforest. R News 2, 3 (2002) 18-22.

Cite this article as: Wehr R. Fuchs A. \& Breuss S. 2021. Statistical tyre/road noise modelling based on continuous 3D texture data. Acta Acustica, 5, 52 . 